\title{
Application of Nanoparticles in Applied Science: A Review
}

\author{
Anand Pal ${ }^{1}$, Sarita Rani $^{2}$, Jagdish Parshad $^{3}$, Tejpal $^{4}$ and Satpal $^{5}$ \\ ${ }^{1}$ Department of Senior Secondary Education, ${ }^{2}$ Department of Agronomy, \\ ${ }^{3}$ Department of Microbiology, ${ }^{4}$ Department of Zoology, ${ }^{5}$ Department of Forage Section, \\ Genetics and Plant Breeding, CCS Haryana Agricultural University, \\ Hisar, Haryana, India-125004 \\ *Corresponding author
}

\section{A B S T R A C T}

Keywords

Nanoparticles, Nanotechnology, Agriculture

Article Info

Accepted:

17 September 2019 Available Online:

10 October 2019
Nanoparticles have wide applications in everyday life. As world population is increasing, it is necessary to use the modern technologies to fulfill needs of present era. Nanoparticle based technology is one of them and has many applications in all stages of production, processing, storing, packaging transports, precision farming techniques, in almost all consumer products, enhancing the ability of plants to absorb nutrients, more efficient and targeted use of inputs, disease detection and control diseases, withstand environmental pressures, in all the fields like cosmetics, paint industry, in medicine, in medical treatment, in food industry, horticulture, agriculture, materials for construction, environmental safety, energy source and in energy conservation. We can make new devices at low cost and more useful to society. Nanoparticle based technology has entered in everyday life and made the life very easy.

\section{Introduction}

Nanoparticles are the particles having size in between 1 to 100 nanometers and are classified into compact materials and nanodispersions. The compact material includes nanostructured materials i.e., materials isotropic in the macroscopic composition and consisting of contacting nanometer-sized units as repeating structural elements (Gusev and Rampel, 2004). The particles with small size in the range from a few to several tens of nanometers are called quasi zero-dimensional mesoscopic systems, quantum dots, quantized or Qparticles, (Khairutdinov et al., 1996). Nanoparticles can differ in their basic properties from other particles from long and well-known ultra dispersed powders with a 
grain size above $0.5 \mu \mathrm{m}$. As a rule, nanoparticles are shaped like spheroids due to ordered arrangement of atoms (or 2 ions) i.e. nanocrystallites.

Nanoparticles with discrete electronic energy levels are referred to as quantum dots or artificial atoms; used in typical semiconductor and magnetic materials.

Many magnetic nanoparticles have the same set of electronic levels and are of great scientific interest because they represent a bridge between bulk materials, molecules and structures at an atomic level.

The term cluster widely used in the chemistry literature in previous years, is currently used to designate small nanoparticles with sizes less than $1 \mathrm{~nm}$. The magnetic molecular clusters (Magnetic polynuclear coordination compounds) belong to the special type of magnetic materials with unique magnetic characteristics, which have the distributions in sizes and are the fully identical small magnetic nanoparticles.

Their magnetism is usually described in terms of exchange-modified paramagnetism (Gubin 2009) and; has wide application in biology, chemistry, environmental studies, medicine, and biotechnology and has strong impact on society. The potential uses of nanoparticles in the environmental sector include waste water management, contaminated soil treatment, sensors and energy storage.

\section{Types and classification}

Nanomaterials are classified different categories based up on materials used, dimensions of electrons movements, origin, fabrication methods and characteristics. These are categories into four types i.e. carbon based, inorganic, organic and composite nanoparticles. These are discussed below one by one:

\section{Carbon-based nanoparticles}

The nanoparticles which contain carbon, are found in spheres, hollow tubes, or ellipsoids. It includes Fullerenes $\left(\mathrm{C}_{60}\right)$, carbon nanotubes (CNTs), carbon nanofibers, carbon black, graphene (Gr), and carbon onions. These are produced by Laser ablation, arc discharge and chemical vapour deposition (CVD) methods in which carbon-based materials (except carbon black) are used.

\section{Inorganic-based nanoparticles}

These are made up of metal and metal oxides and are synthesized from metals like $\mathrm{Ag}, \mathrm{Au}$, $\mathrm{Si}$, metal-oxides viz. $\mathrm{ZnO}$ and $\mathrm{TiO}_{2}$. These are used in semiconductors and ceramics.

\section{Organic-based nanoparticles}

These are made up mostly from organic substances or compounds, excluding carbonbased or inorganic-based nanomaterials. The utilization of properties of non-covalent interactions for the self-assembly and; design of molecules helps to transform the organic nanomaterials into many desired structures such as micelles, dendrimers, liposomes and polymer of nanoparticles.

\section{Composite-based nanomaterials}

These nanomaterials are multiphase nanoparticles and nanostructured materials with one phase on the nanoscale dimension that can either combine one nanoparticles with other one or nanoparticles combined with larger or with bulk-type materials lead to form hybrid nanofibers or more complicated structures like metal-organic frameworks.

The composites may be any combinations of carbon-based, metal-based, or organic-based nanomaterials with any form of metal, ceramic, or polymer bulk materials. 
Classification of nanomaterials based on their dimensions

Nanomaterials can also be classified on the basis of electron movement along with the dimensions i.e. electrons in zero dimension nanomaterials are trapped in a dimensionless space whereas as one dimension nanomaterials have electrons with moving ability along the $\mathrm{x}$-axis. Similarly, two and three dimension nanomaterials have movement of electron along the $\mathrm{x}-\mathrm{y}$-axis, and $\mathrm{x}, \mathrm{y}, \mathrm{z}$-axis, respectively.

\section{Classification of nanomaterials on the basis} of origin

On the basis of their origin of materials used in the preparation of nanomaterials, nanoparticles are classified as natural or synthetic nanoparticles.

\section{Natural nanomaterials}

These are produced in nature either by biological species or through anthropogenic activities. These are produced by the substances available in the natural resources present on earth.

\section{Synthetic nanomaterials}

These nanomaterialsare produced by physical, chemical, biological or hybrid methods using mechanical grinding, engine exhaust and smoke. These are also called as engineered nanomaterials.

\section{Applications of nanoparticles in different fields}

\section{In Medicine}

In recent technique nanoparticles are used to transport drugs at specific target and to particular cells. They can be used to supply heat, light and other substance in particular area that helpful in direct treatment of diseased cell. This technique can be very helpful in treatment of cancer cells (P Bradley, 2011). The use of polymeric micelle nanoparticles to deliver drugs to tumours: The use of polymer coated iron oxide nanoparticles to break up clusters of bacteria, possibly allowing more effective treatment of chronic bacterial infections. The surface change of protein filled nanoparticles has been shown to affect the ability of the nanoparticle to stimulate immune responses. These nanoparticles may be used in inhalable vaccines. The cerium oxide nanoparticles act as an antioxidant to remove oxygen free radicals that are present in a patient's bloodstream following a traumatic injury. The nanoparticles absorb the oxygen free radicals and then release the oxygen in a less dangerous state, freeing up the nanoparticle to absorb more free radicals. Researchers are developing ways to use carbon nanoparticles called nanodiamonds in medical applications. For example, Nanodiamonds with protein molecules attached can be used to increase bone growth around dental or joint implants.

\section{In agriculture and horticulture}

World agriculture is facing the major challenges like changing climate, sustainable use of natural resources, urbanization, environmental hazards like runoff and accumulation of herbicides, pesticides and fertilizers etc. These issues are further intensified by an increase in food demand that will be needed to feed an estimated population of 6-9 billion by 2050 (Chen and Yada, 2011). Many technologies have been developed by researchers that have the potential to enhance farm productivity and also decrease the environmental and resource costs which are related with agricultural production (Ditta, 2012). Numerous reports have revealed the use of nano-particles in agriculture such as 
nano scale carriers for efficient delivery of herbicides, pesticides, plant growth regulators and fertilizers etc., nano encapsulation for reduced use of pesticides. Similarly, use of nano herbicides in an eco-friendly way, without leaving toxic residues in environment, is a better approach for eradication of weeds with reduced amount of herbicides.

Nano-particles can also be used as disinfectants in packaging and engineering of food to increase the shelf life of food products (Ali et al., 2014).Nanotechnology has potential to facilitate the future stage of precision farming techniques and this will enhance agricultural potential in getting higher yields in eco-friendly way even in present day challenging environment (Sugunan and Dutta, 2010).

\section{In manufacturing and materials}

Ceramic silicon carbide nanoparticles dispersed in magnesium produce a strong, lightweight material. A synthetic skin that may be used in prosthetics has been demonstrated with both self-healing capability and the ability to sense pressure. The material is a composite of nickel nanoparticles and a polymer. If the material is held together after a cut it seals together in about 30 minutes giving it a self-healing ability.

Also the electrical resistance of the material changes with pressure, giving it sense ability like touch. Silicate nanoparticles can be used to provide a barrier to gases (for example oxygen), or moisture in a plastic film used for packaging. This could slow down the process of spoiling or drying out in food. Zinc oxide nanoparticles can be dispersed in industrial coatings to protect wood, plastic and textiles from exposure to UV rays. Silicon dioxide crystalline nanoparticles can be used to fill gaps between carbon fibres, thereby strengthening tennis racquets. Silver nanoparticles in fabric are used to kill bacteria, making clothing odour-resistant.

\section{In energy and electronics}

Researchers have used nanoparticles called nanotetrapods studded with nanoparticles of carbon to develop low cost electrodes for fuel cells. This electrode may be able to replace the expensive platinum needed for fuel cell catalysts. Researchers at Georgia Tech, the University of Tokyo and Microsoft Research have developed a method to print prototype circuit boards using standard inkjet printers. Silver nanoparticle ink was used to form the conductive lines needed in circuit boards. Combining gold nanoparticles with organic molecules creates a transistor known as a NOMFET (Nanoparticle Organic Memory Field-Effect Transistor). This transistor is unusual in that it can function in a way similar to synapses in the nervous system. A catalyst using platinum-cobalt nanoparticles is being developed for fuel cells that produce twelve times more catalytic activity than pure platinum. In order to achieve this performance, researchers anneal nanoparticles to form them into a crystalline lattice, reducing the spacing between platinum atoms on the surface and increasing their reactivity. Researchers have demonstrated that sunlight, concentrated on nanoparticles, can produce steam with high energy efficiency. The "solar steam device" is intended to be used in areas of developing countries without electricity for applications such as purifying water or disinfecting dental instruments.

A lead free solders reliable enough for space missions and other high stress environments using copper nanoparticles. Silicon nanoparticles coating anodes of lithium-ion batteries can increase battery power and reduce recharge time. Semiconductor nanoparticles are being applied in a low temperature printing process that enables the 
manufacture of low cost solar cells. A layer of closely spaced palladium nanoparticles is being used in a hydrogen sensor. When hydrogen is absorbed, the palladium nanoparticles swell, causing shorts between nanoparticles. These shorts lower the resistance of the palladium layer.

\section{In electro deposition}

Nanostructured materials can also be produced by electro deposition. These films are mechanically strong, uniform and strong. Substantial progress has been made in nanostructured coatings applied either by DVD or CVD. Many other non-conventional processes such as hypersonic plasma particle deposition (HPPD) have been used to synthesize and deposit nanoparticles. The significant potential of nanomaterial synthesis and their applications is virtually unexplored. They offer numerous challenges to overcome. Understanding more of synthesis would help in designing better materials. It has been shown that certain properties of nanostructured deposits such as hardness, wear resistance and electrical resistivity are strongly affected by grain size. A combination of increased hardness and wear resistance results in a superior coating performance.

\section{In semiconductor physics}

By using nanoparticles we can decrease power consumption and Size of semiconductor devices. Storage capacity of memory chip can also be increased. We can improve quality of integrated circuit.

\section{In Domestic product}

Nanoparticles can be used in many domestic products in every day used by using nanoparticles in cosmetic products we can supply vitamins deep into the skin. By using carbon nanoparticles we can make fire resistant furniture. Quality and storage of lithium ion battery can be much improved with the help of nanoparticles. By using some nano composite plastic we can make scratch resistant body of vehicles and other appliances. We can make light weight, rustproof and strong long life of body of appliance. Nan crystalline coated silver can be used to kill the bacteria in very short time. Chemical-Free [sic] Sunscreen SPF 15 (Burts BeesR,Inc.) contains nano-sized particles of titanium dioxide as the active ingredient. Food Supplements and food storage: Mesozinc nutritional supplement containing 30 parts per million(ppm) zinc nanoparticles are used to store food.

\section{In food storage}

Food storage containers are infused with silver nanoparticles as they are antibacterial agent so protect from bacteria. Deodorizer--"silver nano poly system" acts as an antibacterial and deodorizer. Appliances: Samsung Washing Machine)-Silver nano technology "can be used to sterilizes the clothes. Vacuum Cleaner nano-silver coated cyclone canister removes bacteria. Air Conditioner contains silver nano filter and silver nanoevaporator to purify the air. Clothing: Sport Anklet Socks silver Active-- treated with nanoparticles of silver (typically $25 \mathrm{~nm}$ ) as bactericide and fungicide.

Coatings: Pilkington Active Self Cleaning Glass-glass coating that works with ultraviolet (UV) light and rain to keep glass free from organic dirt. UltimaR Photo Paper (Eastman KodakR Company) nine-layer composition incorporates ceramic nanoparticles to resist the effects of heat, humidity, light and ozone gas. Electronics and computers: Invisicon (EikosR) Invisicon ink used to create transparent conductive coatings and manufacture printed circuits on transparent plastic films; Microprocessor chip manufactured by IBM using IBM's 90 
nanometer Silicon on Insulator (SOI) technology to reduce heat and improve performance.

\section{In production of nanoadsorbent}

Pharmaceuticals city wastes are the main pollutants in drinking water. Nanoparticles can be used to purify the drinking water in mainly three types as adsorbent, as catalyst and as membrane to remove pollutants from water. Adsorption can remove organic and inorganic pollutants from waste water. Nanoadsorbant provide large area and large number of active sites compared to normal adsorbent. So nanoparticles are very helpful in wastewater treatment. nanoadsorbent may be magnetic, non magnetic and carbon based and may be metal oxide and zeolites. Nanoparticles used as adsorbent should be no harmful and moreadsorption.

\section{In Water treatment}

In water treatment, removal of nonbiodegradable organic pollutants is not possible by conventional treatment methods. So, to find a new, efficient and eco-friendly technology that can remove these pollutants with less use of energy and chemicals is the need of present day.

Therefore, researchers have concentrated on some advanced alternative methods of oxidation that have capability of oxidizing and mineralizing the organic chemicals (Comninellis et al., 2008).

To improve the biodegradability of organic contaminants and to remove the recent microbial pathogens, Photo catalysis has been considered as best method. Photocatalytic oxidation consists of a class of reactions that use the catalyst activated by solar or other forms of energy (Bahnemann 2004)

\section{In production of Nanomembrane}

Nanomembrane can be used in waste water treatment because they have low cost and low power consumption. Rao (2014) found that for improving the water quality of desired value, the pressure driven treatment of wastewater has been proved best in this technique.

\section{In Remediation}

No chemical addition is required in water remediation because of its more separation efficiency and easy operation. It does not lead to secondary pollution as well as no regeneration of spent media is required (Balamurugan et al., 2011). The performance of the membrane system is dependent on the membrane material, which depend on membrane selectivity and permeability.

The common membrane materials applied for water treatment are polymers, cellulose acetate, poly acrylonitrile, and polyamide (Yang et al., 2009). Based on the pore size and filtration application, the membrane process can be classified as microfiltration for suspended solids, Protozoa, and bacteria removal, ultrafiltration for virus and colloid removal, nanofiltration for hardness, heavy metals, and dissolved0organic matter removal.

\section{In various energy sources}

Nano particles have great potential to increase energy efficiency, energy conservation, storage usage and saving. Nanoparticles can used to increase the efficiency of solar cell and fuel cell. Solar cell or photovoltaic cell is the device that converts solar energy in to electrical energy. They are made of semiconductor silicon or germanium. They have efficiency about $14 \%$ and high cost. The first generation solar cells are made of silicon wafer having performance $15 \%$ to $20 \%$. The second generation solar cells are made on the 
basis of thin film coating using amorphous silicon and cadmium telluride having much less cost while third generation cells are based on nanoparticles and nano-porous materials but they are in research stage.

\section{In artificial photosynthesis}

Artificial photosynthesis process is important means of renewable energy production with use of fossil fuels and carbon emission. Nanotechnology helps in this process

\section{In Energy storage batteries}

In almost all the electronics devices in small power tools and electric vehicles, lithium ion batteries are used. For more safety anode may be used made of nanowires or nanorod materials. In latest discoveries ultra high capacity anode materials such as Si nanowires, Ge nanowires and tin nanoparticles coated with carbon embedded with grapheme has more cycle performance and high conductivity

\section{In production of Super capacitors}

Super capacitor is ultra capacitor they can store more energy electrostaticaly by polarizing the electrolyte. The performance of super capacitor is between normal capacitor and batteries.

The most remarkable property of super capacitor is high power density excellent reversibility quick operation and long life.

\section{In Pollution control}

Nanoparticles plays important role in decreasing pollution by using raw materials. Some nanowires of potassium manganese oxide were recently observed for cleaning up oil spills and other organic pollutants. $\mathrm{TiO}_{2}$ can be used to breakdown of nitrous oxide and other pollutants into less harmful substances.

\section{In carbon capture}

Fossil fuels are used by most of the industries through combustion and release carbon dioxide in atmosphere by using nanoparticles amine based absorption. The adsorbent like activated carbon and zeolite being high thermal and chemical stability can be used as carbon dioxide capture.

\section{In environmental sensing}

Nanoparticles can be used to build low cost high sensitive detection system for monitoring air and water quality. These nanoparticles based sensor have ability to detect toxins, heavy metals and organic pollutants at very low concentration.

\section{In Sensors for heavy metal ion detection}

Heavy metals such as $\mathrm{Cd}$, lead, $\mathrm{Hg}$, As, are released in industrial waste they are highly toxic even at very small levels they are threat to human beings and all living beings the use of nanoparticles have improve the performance of sensor and wide range of detection principle like optical, electrical, ion exchange, semiconductor metal oxide conductive polymers.

\section{In optical sensing}

Nanoparticles are used in optical sensing this include fluorescent, colorimetric, surface Plasmon resonance and surface enhanced Raman scattering sensors.

\section{In electro chemical sensing}

In addition to heavy metal ion detection single walled nanotubes were used to develop sensor for sensitive detection of microcystin - LR similarly other sensors for bacterial detection include mannoseen capsulated gold nanoparticles for the detection of Escherichia coli and detection of cholera toxin. 
Hence it is clear that nanoparticles are widely used in almost all the fields. The use of nanoparticles can make major change in future life; make it easy at low cost but possible environmental, social, and ethical implication should be considered such as testing of toxicity of nanoparticles, safety design of nanomaterials, ecological impact of nanoparticles. Therefore risk of their use in the environment and possible bad effects on living beings needs to be checked. The worse activities of human are disrupting the ecosystem balance by filling the environment with large amounts of hazardous toxicants that pollute the atmosphere especially, soil and water and consequently threaten health of human being and wise and precise use of nanoparticles can help in making balance in ecosystem with lots of other benefits like reducing cost, ease of working etc.

\section{References}

Ali M.A., Rehman I, Iqbal A, Din S, Rao AQ, Latif A, Samiullah TR, Azam S, Husnain T. (2014). Nanotechnology, a new frontier in Agriculture. Adv. life sci., 1(3), pp. 129-138.

Bahnemann (2004) https://www. research gate.net

Balamurugan, A., R. Jayanthi, P. Nepolean. (2011) https://www.sciencedirect.com
Bradley, P. (2011) Everyday Applications of nanotechnology.http://ccweek.com/arti cle-2630-every day- applications-of nanotechnology.html, Retrieved march 2014

Chen H. and Yada R. (2011) Trends Food Sci. Technol. 22: 585

Christos Comninellis (2008) https://online library.wiley.com.

Ditta, A. (2012) How helpful is nanotechnology in agriculture? Advances in Natural Sciences: Nanoscience and Nanotechnology 3(3): 033002.

Gusev, A.I. and Rempel, A.A., (2004). Nanokristallicheskie materialy (Nanocrystalline Materials), https:// link.springer.com.

Khairutdinov, R.F. (1996), Chemistry of Semiconductor Nanoparticles, https:// link.springer.com.

Rao, L.N. (2014) Nanotechnological methodology for treatment of wastewater. Int. J. Chem. Tech. Res., 6 (4), p. 2529

Sugunan A. and Dutta J. (2010). Pollution treatment, remediation and sensing. Nanotechnology. https://onlinelibrary.wiley.com/doi/abs /10.1002/9783527628155.nanotech013

Yu Yang, Stephon, J. Read, Lynnc Miller. (2009)https://doi.org

\section{How to cite this article:}

Anand Pal, Sarita Rani, Jagdish Parshad, Tejpal and Satpal. 2019. Application of Nanoparticles in Applied Science: A review. Int.J.Curr.Microbiol.App.Sci. 8(10): 2318-2325. doi: https://doi.org/10.20546/ijcmas.2019.810.269 